\title{
Issues of Long-Hop and Short-Hop Routing in Mobile Ad Hoc Networks: A Comprehensive Study
}

\author{
M. Tarique, A. Hossain, R. Islam and C. Akram Hossain \\ Dept. of Electrical and Electronic Engineering, \\ American International University-Bangladesh,
} Tel: 880-1745819026Ｅ-mail: tariquemohammed@aiub.edu

Received: June 29, 2010 Accepted: July 14, 2010 DOI: 10.5296/npa.v2i2.430

\begin{abstract}
One of the fundamental problems of Mobile Ad hoc Networks (MANETs) is to determine whether it is advantageous to route packet over many short-hops or a few long-hops. Short-hop and long-hop routing issues have been investigated in the literatures since the early days of MANETs. Maximization of network throughput was the main focus of these works. But the maximization of network throughput is not the only issue of MANETs. There are other important issues of concern including overhead control packets, energy consumption, connectivity, shadowing effects and packet delay. All these issues have been investigated in this paper. Network Simulator (NS-2) has been used to create and simulate MANETs to investigate these issues. The main objectives of this paper are as follows (1) to find the merits and de-merits of short-hop and long-hop routing schemes, (2) to come up with a guideline so that one can choose an appropriate routing scheme for MANETs, and (3) to open up new area of researches for further investigations on the issues discussed in this paper.
\end{abstract}

Keywords: Mobile Ad hoc, routing, long-hop, short-hop, energy consumption, mobility, overhead, network life, network

\section{INTRODUCTION}

In the recent years, Mobile Ad hoc Networks (MANETs) are considered as a suitable means of providing instant networking to a group of mobile users. MANETs are self-organizing and self-configuring. No centralized administration is required to operate and maintain such networks. In MANET, mobile nodes communicate with each other in a multi-hop fashion. It means that a source node sends a packet to a destination node via intermediate nodes called hops. Routing protocol helps a mobile node to discover these intermediate hops. One of the fundamental problems of routing protocol is to decide whether a mobile node should use many short-hops or a few long-hops. The length of a hop (or the transmission range) depends on the propagation characteristics and the transmission power level of a mobile node [1]. 
In this paper, the transmission range was varied by adjusting the transmission power of a mobile node. Higher transmission power level means longer hop. On the other hand, low transmission power level means shorter hop.

The long-hop and the short-hop issues have been investigated by the researchers since the early days of packet radio network [2]. An approximate analysis in [3] shows that the transmission range of a mobile node should be small, but not too small so that there may occur network partitioning (i.e,all mobile nodes in a network may not be reachable). Once a network is partitioned, the normal operation of the network may be seriously affected. Another analysis in [4] shows that there is a trade-off between the transmission range of a mobile node and the network throughput. Higher transmission power level decreases network throughput. On the other hand, lower transmission power level increases network throughput. The analysis presented in [4] has also shown that a mobile node should adjust its transmission power to a level so that it should have at least six neighbors. An extension of this work [5] concludes that a mobile node should have eight neighbors. A similar work [6] has proved that there is a critical transmission range for a mobile node, which is just enough to maintain the network connectivity. Mobile nodes should use this critical transmission range. Another related work [7] shows that a mobile node should adjust transmission power to maximize the node's battery life. According to this scheme mobile nodes form groups called 'clusters'. In a cluster, each member node should adjust its transmission range to reach the furthest node located in the same cluster. One common limitation of all these works is that the mobile nodes are assumed to be static. Mobility has been considered in [8]. In this work, the author claimed that the transmission range of a mobile node should be adjusted to maximize the number of packets that can be delivered to the destinations under mobility a condition. Simulation results presented there in show that the number of neighbors should be increased if there is a high mobility condition in a network. But there is no optimum number of neighbors that can maximize the packet delivery. A recent work [9] shows that there are 18 cases where long-hop routing is advantageous over short-hop routing. The authors' claims are based on the laboratory experiments with a small network consisting of only 10 sensor nodes. For this kind of small network, it is hard to judge whether the short-hop or the long-hop routing is better. Because it is shown in the literature [10] that the performance of a routing protocol varies widely with the network size. In [11], the power control problem is viewed as a network layer problem. The COMPOW protocol is proposed in this paper. According to the COMPOW protocol each node has to adjust its transmit power such that its connectivity degree (number of one-hop neighbors) is bounded. A transmit power control algorithm proposed in [12] attempts to optimize the average end-to-end throughput by controlling the degree of the nodes. In [13] a distributed topology control algorithm is proposed. The technique is based on the utilization of direction information. The CLUSTERPOW algorithm proposed in [14] aims on the increase of network's capacity by increasing spatial reuse. The algorithm consists of simply using the lowest transmit power level $p$, such that the destination is reachable (in multiple hops) by using power levels no larger than $p$. A new transmission assignment strategy has been proposed in [15]. The authors suggested in this paper that the transmission range should be optimized to reduce energy consumption while network connectivity should be preserved. They also showed that $30 \%$ of energy can be saved if the proposed algorithm is used.

The common limitation of most of the previous works cited so far is that the maximization of network throughput was the main target of investigation. But throughput maximization is not the sole issue for MANETs. There are other important issues that are related to the performance of a MANETs. These issues are as follows: 
a) Overhead control packets generated in a network is an important design issue of an efficient routing protocol. Overhead control packets are generated by different activities of the mobile nodes in a network including the route discovery. These overhead packets occupy bandwidth and may overwhelm a network if not controlled [10].

(b) Energy conservation is another important issue of MANETs. Mobile nodes are usually equipped with limited batteries. In many applications, these batteries cannot be replaced or re-charged, Hence it is imperative that the mobile nodes should be operative as long as possible.

(c) Packet loss is another important issue of MANET. Packet loss can make a network unreliable one. Three main reasons of packet losses in a network are: (1) packet collisions that occur from the simultaneous packet transmissions by a number of neighboring mobile nodes, (2) packet drops due to limited buffer size of a mobile node, and (3) packet loss due to shadowing effect.

(d) End-to-end packet delay is another important design parameter of MANETs especially for delay constraint applications. The end-to-end packet delay depends on the traffic intensity as well as the number of hops that a packet travels from a source to a destination in a given network. Hence the end-to-end packet delay depends on the transmission range of a mobile node too.

(e) Node mobility affects routing decision as well as packet losses in MANET. High mobility increases route 'breakage' rate and hence increases packet losses.

(f) An efficient medium access control mechanism is very essential for MANETs. IEEE 802.11[18] is considered as a popular choice for medium access control scheme in MANET. The Medium Access Control (MAC) protocol like IEEE 802.11 also generates a huge number of special types of control packets namely Request-to-Send (RTS), Clear-to-Send (CTS), and Acknowledgement (ACK), .

(g) Shadowing effects [1] are considered another problem of MANET. Shadowing effect explains why the signal level varies with time about a mean value for a given transmitter and receiver distance. Hence there is always a probability that a packet will be received at a receiver with a signal level that is less than a threshold level to cause packet loss.

Although there are other important issues of MANETS that are related to the transmission range of a mobile node, we focus only on the above mentioned issues by limiting this work within a manageable size. In order to investigate all these issues of long-hop and short-hop routing, the Dynamic Source Routing (DSR) [17] protocol has been chosen as the routing protocol for the networks. Because the DSR protocol is considered as one of the most popular routing protocols extensively investigated in the literatures. While it is likely that the network performances will vary with the routing protocol used, the results obtained with DSR protocol can be generalized to most on-demand ad hoc routing protocols. A brief description of DSR protocol has been provided in the next section. The rest of the paper is organized as follows. Section 3 contains the issues investigated in this paper including simulation results. Section 4 presents a guideline for the network designer that will help them to choose an appropriate routing scheme between short-hop or long-hop routing. Section 5 concludes this paper. This concluding section also contains the future research directions related to this work.

\section{THE DYNAMIC SOURCE ROUTING (DSR) PROTOCOL}

The DSR protocol consists of two main mechanisms: (1) route discovery, and (2) route maintenance. Route 
discovery is the mechanism by which a source node discovers a route to a destination. During a route discovery process, a source node initiates the route discovery by broadcasting a request message to its neighbors. When the neighboring nodes receive the request packet, they add their addresses in the request packet and re-broadcast that request message to their neighbors. This process goes on until the request packet is received by a destination node. A route discovery mechanism is illustrated in Fig. 1(a). In this figure, node $\mathrm{A}$ is attempting to discover a route to node $\mathrm{E}$. To initiate the route discovery process, node A transmits a 'route request' packet as a single local broadcast packet, which is received by all nodes currently within the transmission range of A including node B. Each route request packet identifies the initiator and the target of the route discovery, and also contains an unique request identification determined by the initiator of the request. Each route request also contains a listing of the addresses of the intermediate nodes through which this particular copy of the route request packet has been forwarded. When another node receives this route request (i.e., node B in this example), if it is the target of the route discovery, it returns a 'route reply' to the initiator of the route discovery process, giving a copy of the accumulated route record from the route request packet. When the initiator receives this route reply, it records this route in its route cache for use in sending subsequent packets to this destination. Otherwise, if this node receiving the route request has recently seen another route request message from this initiator bearing this same request identification and target address or if this node's own address is already listed in the route record of the route request, this node discards the request. Otherwise, this node appends its own address to the route record in the route request and propagates it by transmitting it as a local broadcast packet (with the same request identification). In this example, node $\mathrm{B}$ re-broadcasts the route request, which is received by node $\mathrm{C}$; nodes $\mathrm{C}$ and $\mathrm{D}$ each also, in turn, re-broadcast the request, resulting in the request packet being received by node $\mathrm{E}$.

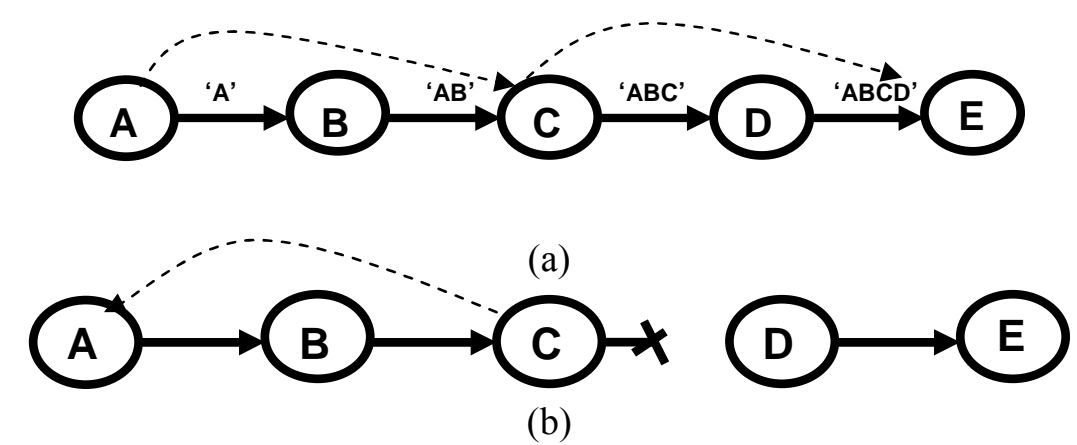

Fig.1 (a) Route discovery, and (b) Route maintenance of DSR protocol

Route maintenance is a mechanism by which a node is able to detect changes in the network topology. While originating or forwarding a packet using a source route, each node transmitting the packet is responsible for confirming that a data packet can travel over the link from that node to the next hop. For example, in the network scenario shown in Fig. 1(b) node A has originated a packet for node E using a source route through intermediate nodes B, C, and D. In this case, each node is responsible to monitor the link between itself to the next hop. For example, node A is responsible for the link from A to B, node B is the responsible for the link from $\mathrm{B}$ to $\mathrm{C}$ and so on. An acknowledgement can provide confirmation that a link is capable of carrying data, and in wireless networks, acknowledgements are often provided by an existing standard part of the MAC protocol such as IEEE 802.11 [18]. In this example, when node C detects that the link between itself to node $\mathrm{D}$ is broken, node $\mathrm{C}$ creates a route error message and sends that packet to node A. After receiving the route error message, node A marks the route as 'invalid' in the route 
cache and tries to find an alternative route to the destination node E. If no such route is found in the route cache, node A initiates a new route discovery process.

The basic route discovery and the route maintenance operations of the DSR protocol mentioned above depend on a fundamental question. What will be the transmission range of a mobile node? If a higher transmission range were used, the source A could have discovered a path to the destination D in fewer hops. For example, the new route could be A-C-E as shown by the dashed line in Fig.1(a). Hence the route discovery time could have been shortened. If a routing path of few hops is used, there is a less probability to break that path because of node movement, battery exhaustion and other causes. On the other hand, if a route contains many hops, there is more likely to have a route breakage. Another advantage of using long-hop routing is that a packet will travel a few number of hops and hence the end-to-end delay of a packet will be reduced too. The route maintenance operation also depends on the transmission range of a mobile node. Higher transmission range can expedite the route maintenance operation. For example, the route maintenance operation illustrated in Fig. 1(b) would have been a quick one if node C could directly send route error message to source A. This kind of quick route maintenance operation can save packet loss in a network. Based on these observations of basic route discovery and route maintenance operation one may be tempted to set a higher transmission range of a mobile node. But it may not always be wise to choose a higher transmission range. Because other performances of a network such as overhead packet generation, energy consumption and interference level are also directly related to the transmission range of a mobile node which are explained in the following section.

\section{ISSUES OF LONG-HOP AND SHORT-HOP ROUTING}

Unlike traditional cellular network, an ad hoc wireless network is characterized by peer-to-peer communication, distributed networking, multi-hop routing, energy constraint, dynamic topology and unreliability. Not all of these characteristics are equally important, but depend on specific application. A mobile node transmits a signal directly to any other node. While transmitting signal, mobile node should maintain its transmission power at a proper level so that even if the signal is attenuated, the receiving mobile node can successfully detect a packet. A proper level of transmission power is also important because a high transmission power level increases Signal-to-Interference plus Noise power Ratio (SINR). On the other hand, low transmission power reduces SINR. Low transmission signal level will be attenuated quickly as it travels from one mobile node to another mobile node. Hence transmission power level (i.e., the transmission range) should be carefully selected. The need for transmission range adjustment is illustrated in Fig. 2. In this figure node $n_{1}$ is sending packets to node $n_{2}$ and node $n_{3}$ is sending packet to node $\mathrm{n}_{4}$ simultaneously. The transmission radii of nodes $\mathrm{n}_{1}$ and $\mathrm{n}_{2}$ are shown in this figure by dashed line. Two communications will be successful if none of these two transmissions interferes with each other as shown in Fig. 2(a). Two unsuccessful transmissions are shown in Fig. 2(b). In this case the transmission ranges of node $n_{1}$ and node $n_{3}$ are too high to interfere with each other. Two unsuccessful communications due to very low transmission range are shown in Fig. 2(c). In this case, the signal level is attenuated quickly and node $n_{2}$ and $n_{4}$ fail to detect the packets successfully. Based on the operation of a simple network shown in Fig. 2, we can conclude that transmission power level should not be too high or too low. But it should be maintained at an appropriate level to ensure successful packet transmission. In this paper, we investigated the effects of long-hop and short-hop on the network performances such as network throughput, shadowing effects, energy consumptions, MAC overhead, network connectivity, routing overhead, end-to-end delay, packet loss and mobility which are explained in the following sub sections. 

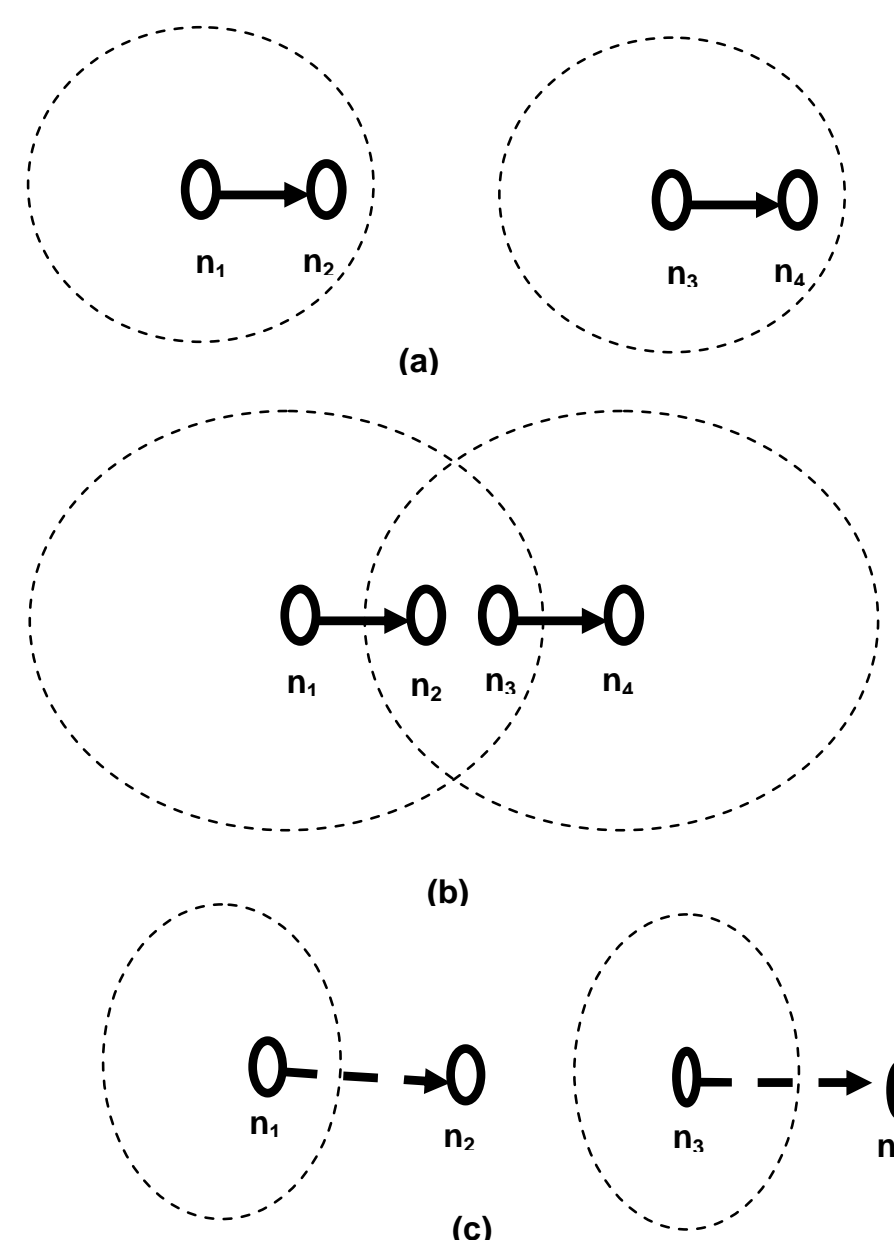

(b)

(c)

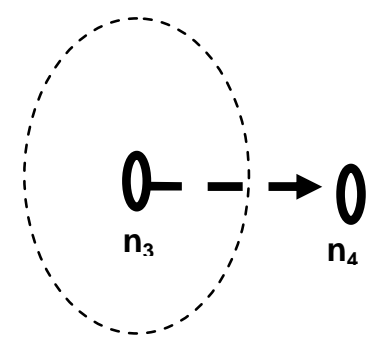

Fig. 2 (a) Two successful communication, (b) unsuccessful communication due to high power, and (c) unsuccessful communication due to low power

\subsection{Network Throughput}

Although the interference level in the simple network shown in Fig. 2 is not significant, the level of interference in a network becomes severe and hence adversely affects the network throughput as the network gets larger. The level of interference in a network is limited by two main factors (1) node density, and (2) traffic intensity. Node density determines the number of nodes located in a given region. If the node density if higher, there will be higher the interference level. Traffic intensity depends on the number of connections set up in a network and it also depends on the packet generation rate associated with each connection. Medium Access Control (MAC) protocol like IEEE 802.11 plays an important role to reduce interference level in a network. IEEE 802.11 uses Carrier Sense Multiple Access with collision Avoidance (CSMA/CA) technique. According to this technique, one mobile node is allowed to transmit a packet at a time for a given region called 'contention region'. In a contention region, a mobile node senses the medium to determine whether it is free or busy. If the medium is free, a mobile node transmits its packet. Other mobile nodes in this region are not permitted to transmit at this moment, but they defer their transmissions for random back-off periods. At the end of this back-off period a mobile node again senses the medium. If it finds that the medium is free, it transmits its packet. This kind of medium access control 
mechanism is illustrated in Fig. 3. In this scenario, mobile nodes A, B, C and E are within the transmission ranges of each other. Hence they form a contention region as shown by the dashed line. In this contention region, only one node is allowed to transmit its packet at a given time. When node A transmits, all other nodes including $\mathrm{B}, \mathrm{C}$ and $\mathrm{E}$ defer their transmissions for random back-off periods. The contention region varies with the transmission range of a mobile node. For a given node density, low transmission range will form smaller contention region. Hence there will be less number of nodes that will try to get access to the medium at a given time and hence mobile nodes will be able to send packets without keeping them in the buffer for a longer period of time. This short period of waiting time of a packet in the buffer reduces packet delay. The contention level of a contention region also depends on the traffic load intensity in that region. When a mobile node has to handle more traffic in a given period of time, it needs to get access to the medium more frequently. Hence contention level increases with the increase of traffic load. By controlling the transmission range of a mobile node the contention level due to traffic intensity and node density can also be controlled. Hence delay per packet will be reduced and the network throughput will be improved.

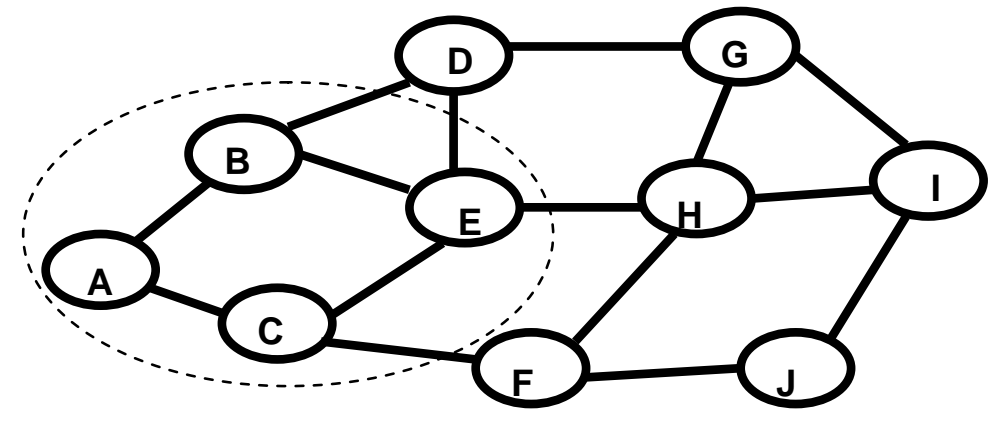

Fig.3 Contention region of a network

In order to investigate the network throughput under different transmission ranges, a network consisting of 200 nodes was created and tested via Network Simulator (NS-2) [19]. The mobile nodes were placed randomly over an area of $1000 \mathrm{~m} \times 1000 \mathrm{~m}$. Ten connections were set-up in the network. Constant Bit Rate (CBR) packet generator was used to generate traffic. IEEE 802.11 was used as MAC layer. The transmission range was $250 \mathrm{~m}$. Ten different topologies were created by using random number generator. Each topology was simulated for 250 second (i.e., simulator time). The results of these simulations were then averaged. The initial packet generation rate was 2.0 packets/second. Then the packet generation rate was increased to $3.0,4.0,5.0,6.0,7.0$ and 8.0 packets/second to increase the traffic intensity level in the network. The simulations were then repeated for lower transmission ranges (i.e., $200 \mathrm{~m}$ and $150 \mathrm{~m}$ ). Network throughput was measured as kilo bits per second (kbps). The results are presented in Fig.4. The most important unique conclusion of this figure is that a network has a maximum capacity (or throughput) limit. After reaching a maximum value the network throughput decreases rapidly. For example, the maximum network throughput was $147 \mathrm{kbps}$ for the transmission range of $250 \mathrm{~m}$. This maximum throughput was achieved at the packet generation rate of 5.0 packets/second. When the packet generation rate was further increased, the network throughput decreased rapidly. Fig. 4 also shows that the network throughput was reduced to $96 \mathrm{kbps}$ at the packet generation rate was 6 packets /second and the network throughput reaches a minimum value at the packet generation rate of 8 packets/second. Hence the network throughput was reduced by $61 \%$ as the packet generation rate was increased from 5 packets/second to 8 packets/second. The transmission range of a mobile node was then reduced to $200 \mathrm{~m}$. At this transmission 


\section{Macrothink}

range the maximum network throughput achieved was $132 \mathrm{kbps}$ at packet generation rate of5 packets/second. The network throughput decreased with the increase in packet generation rate as usual after this point. But the rate of decrease in throughput is less than that of previous case (i.e., when transmission range of $250 \mathrm{~m}$ ). For example, when the transmission range was $250 \mathrm{~m}$, the network throughput was $57 \mathrm{kbps}$ at the packet generation rate of 8packets/second. But the network throughput was $75 \mathrm{kbps}$ at the same packet generation rate when the transmission range was $200 \mathrm{~m}$. It is also depicted from this figure that the maximum throughput was $126 \mathrm{kbps}$ at the transmission range of $150 \mathrm{~m}$. Another important conclusion that can be drawn from Fig. 4 is that the network throughput shows a sustainable performance for higher packet generation rate (i.e., more than 5 packets/second) at low transmission range (i.e., 150 meter). For the highest packet generation rate of 8 packets/second, the network throughput was $118 \mathrm{kbps}$. But for transmission ranges of $250 \mathrm{~m}$ and $200 \mathrm{~m}$, the network throughputs were $57 \mathrm{kbps}$ and 75 kbps respectively at the same rate. We can conclude from Fig.4 that the maximum network throughput was achieved when the transmission range was the highest (i.e., 250 meter). But the network throughput sustains for a longer period of time at lower transmission range.

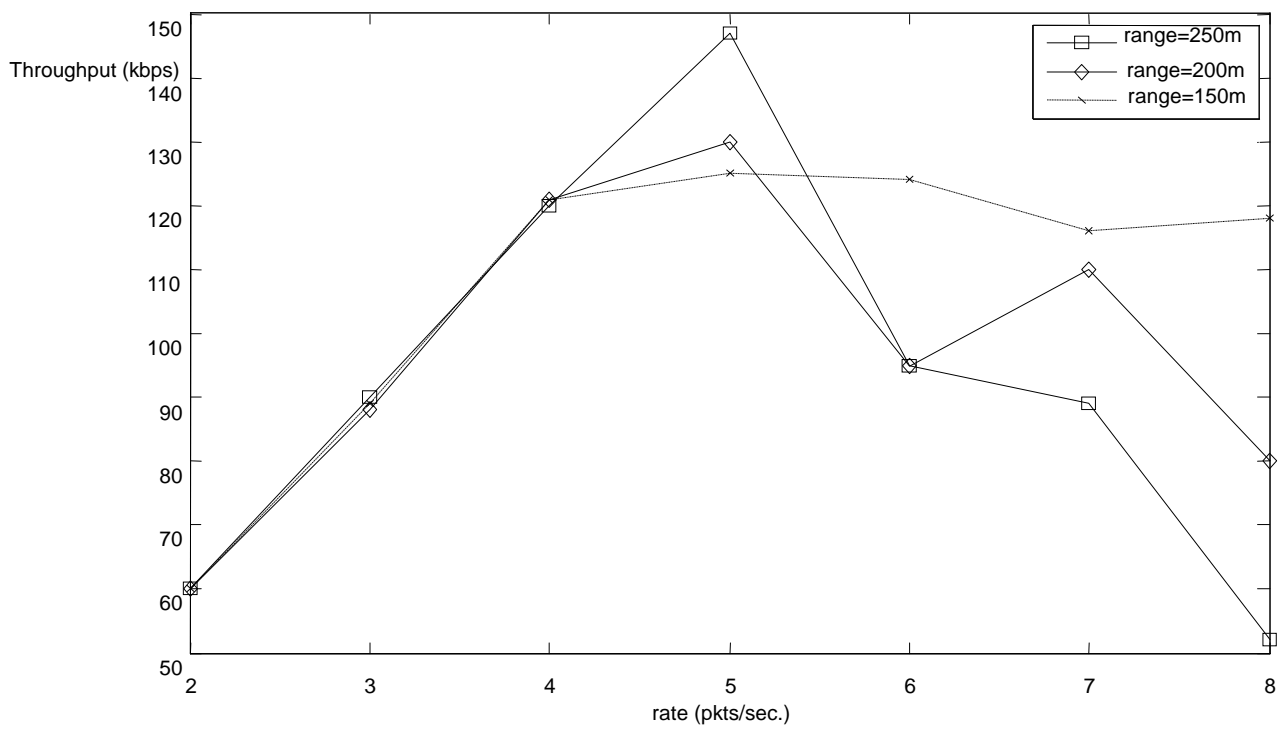

Fig.4 Network throughput comparisons 


\section{Macrothink}

\subsection{Shadowing Effects}

In most practical cases, Signal to Interference and Noise Ration (SINR) varies randomly over time due to the mobility of the nodes, propagation environment and interference characteristics. Measurements have shown that at any value of distance $d$, the path loss $P L(d)$ at a particular location is random and distributed log-normally (in $d B$ ) about the mean distance-dependent value [1]. The path loss is given by

$$
P L(d)[d B]=P L(d)_{\text {mean }}+X_{\sigma}=P L\left(d_{0}\right)_{\text {mean }}+10 n \log \left(\frac{d}{d_{0}}\right)+X_{\sigma}
$$

, where $P L(d)_{\text {mean }}$ is the mean value of path loss at distance $d$ and $X_{\sigma}$ is a zero-mean Gaussian distributed random variable (in $d B$ ) with standard deviation $\sigma$ ( also in $d B$ ) and $\mathrm{d}_{0}$ is the reference distance located in the far-field [1]. This kind of variation of loss around the mean value is called log-normal distribution. The log normal distribution describes the random shadowing effects which occur over a large number of measurements locations that have the same transmitter-receiver separation, but have different levels of clutter on the propagation path. This phenomenon is defined as long-normal shadowing in the literature [1]. Under the log-normal shadowing, the transmission power and the received power are related by the following equation

$$
P_{r}(d)[d B m]=P_{t}[d B m]-P L(d)[d B]
$$

Equation 2 shows that if the transmission power is increased, the received power will increase for a given path loss. Hence the probability that the packet will be successfully received at the destination will increase too.

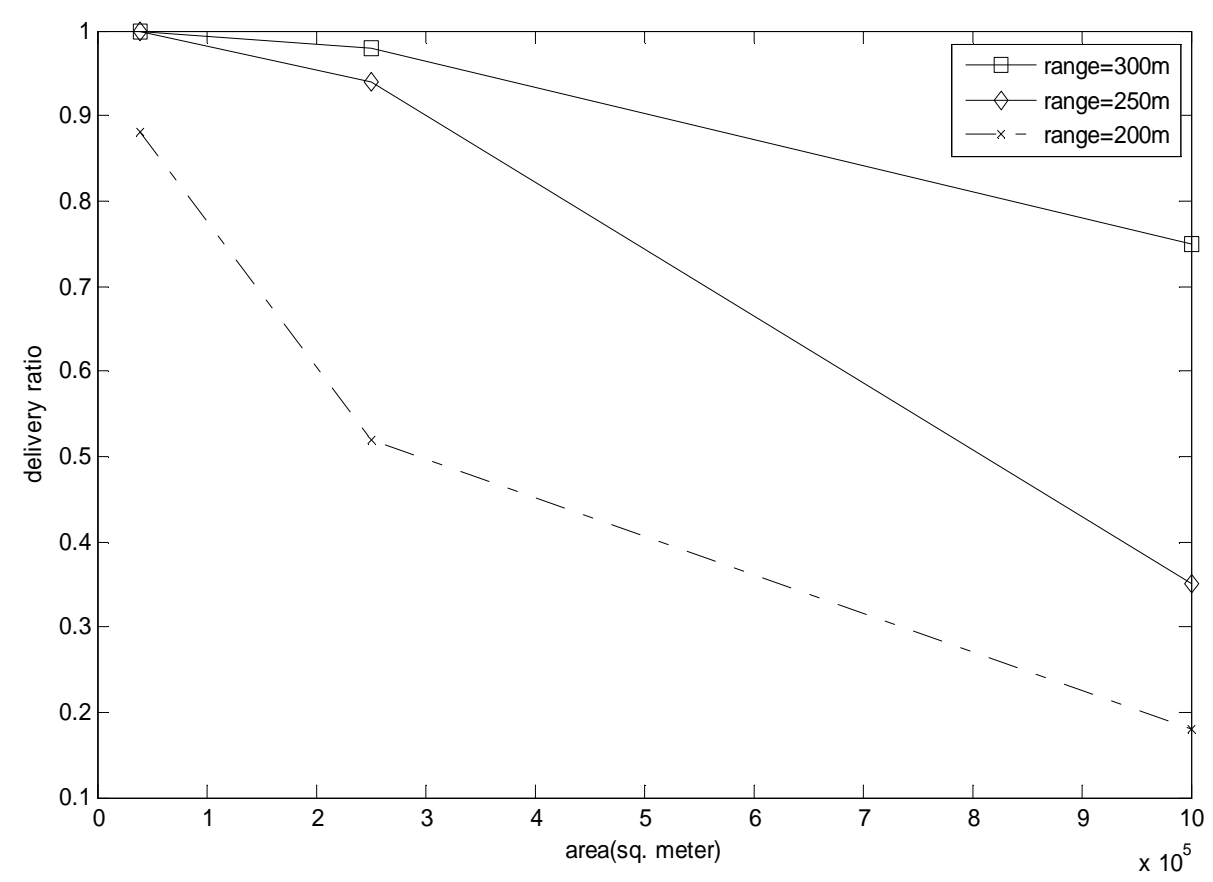

Fig.5 Packet loss due to shadowing effects 
To investigate the shadowing effects, an ad hoc network consisting of 200 nodes was simulated in NS-2. These 200 nodes were placed randomly over an area of $750 \mathrm{~m} \times 500 \mathrm{~m}$. Ten CBR connections were randomly set up in the network. The area of the network was then increased to $1000 \mathrm{~m} \times 500 \mathrm{~m}$ and $1000 \mathrm{~m}$ $\times 1000 \mathrm{~m}$. The number of packets sent and the number of packets received at the destination were used to determine packet losses in the network. The shadowing propagation model available in NS-2 was used with the following parameters: path loss exponent $n$ was 4.0, standard deviation $\sigma$ as $1.0 \mathrm{~dB}$, and the reference distance $\mathrm{d}_{0}$ was 10 meter. Ten different topologies were simulated and the results were averaged for these different topologies. The results of these simulations are depicted in Fig. 5. The figure shows that the packet loss is negligible for a small network and the packet loss increases as the network area increases. The reason is that the length of a link between two mobile nodes increases for a larger network. For example, the delivery ratio was almost $100 \%$ for transmission ranges of $250 \mathrm{~m}$ and $300 \mathrm{~m}$ for a small network (i.e., $750 \mathrm{~m} \times 750 \mathrm{~m}$ ). But transmission range was $200 \mathrm{~m}$, only $10 \%$ packets were lost for the same network. When the network area was increased to $1000 \mathrm{~m} \times 1000 \mathrm{~m}$, the delivery ratio was reduced by $15 \%$ only for the transmission range of 300 meter. For the same network size, the delivery ratio was decreased to $40 \%$ and $20 \%$ as the transmission ranges were set to 250 meter and 200 meter respectively. We can conclude from the results presented in Fig. 5 and also from the signal loss model expressed on Equation 2 that transmission power level should be maintained as high as possible to reduce packet losses due to shadowing effect in the network.

\subsection{Energy Consumption}

Energy constraint may or may not be inherent to all kinds of mobile nodes. Mobile nodes may be attached to a large energy source. However, many mobile nodes are powered by battery of limited capacities. Some of the most exciting applications of MANET fall in this energy constrained category. Mobile node with rechargeable battery must conserve energy to maximize the time between two recharging. In many cases, the batteries of the mobile nodes may not be recharged or replaced as mentioned in Section 3.1. In this paper, energy consumption due to packet transmission is only considered. The energy consumptions by other activities of a mobile node including packet reception have been neglected. The reason is that the energy consumption due to variable transmission power level was the main focus of this study. The energy consumption pattern and energy model in ad hoc networks have been investigated in [20] and [21]. According to these models, the energy spent at wireless node's network card while transmitting a packet is described by the following equation

$$
E\left(D, P_{t}\right)=K_{1} P_{t} D+K_{2}
$$

,where the constant values of $\mathrm{K}_{1}$ and $\mathrm{K}_{2}$ are $4 \mu$-sec/byte and $42 \mu$ Joules respectively. Equation 3 is used as the energy consumption model in this study. In this investigation three types of packets namely data packet, Medium Access Control (MAC) packets and routing packets were considered. The MAC layer packets are namely Clear-to-Send (CTS), Request-to-send (RTS) and Acknowledgement (ACK) packets. The routing packets include route request packets, route reply packets and route error packets. The MAC packets and the routing packets altogether can be called overhead control packets. The number of overhead packets generated in a network depends on the network size and the number of connections set up in the network. When the network size is small, the number of overhead packets is not significant. Hence a small amount 
of node's energy is consumed by overhead packet. On the other hand, if the network size is large, huge overhead packets are generated in a network. Hence a considerable portion of node's energy is spent in transmitting overhead packets. Categorical energy consumptions by different types of packets under varying network size are shown in Fig. 6 . The figure depicts that the energy consumptions by different types of packets depend on the network size. When the network size is small, the most of the energy is consumed by useful data packet. On the other hand the rest of the energy is consumed by overhead (MAC and routing) packets. Fig. 6 also shows that almost $70 \%$ of energy is consumed by useful data packet and the remaining $30 \%$ energy is consumed by overhead packets for a small network. But the overhead packets consume a significant portion of node's energy for larger network. The figure shows that almost $60 \%$ of node energy is consumed by the overhead packet and $40 \%$ of energy is consumed by useful data packet for a network consisting of 300 nodes. The energy consumption is directly related to the transmission power as shown in Equation 3. Hence energy consumption can be reduced if transmission power is reduced. In this study energy consumption per data packet was used as a parameter to determine the energy consumption rate in a network. This is the ratio of total energy consumed by all mobile nodes in the network and total number of data packets delivered to the destinations.

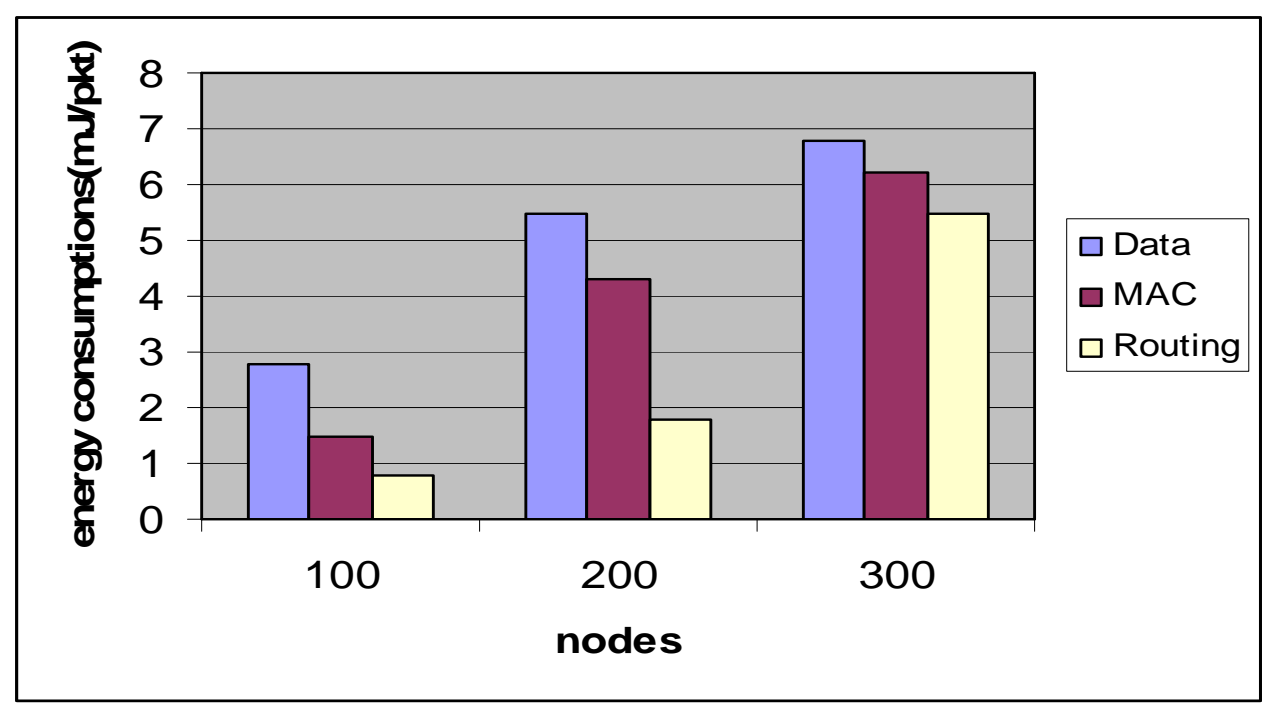

Fig.6 Categorical energy consumptions in ad hoc networks

Typical energy consumption per useful data packet under varying network size is shown in Fig. 7. It is depicted in this figure that the energy consumption per packet increases with the network size. The reason is that a packet travels more hops in a large network. But the energy consumption per packet was the minimum when the transmission range was the minimum (i.e., range is $200 \mathrm{~m}$ ). The energy consumption per packet increases with the transmission range. For example, when network size was 100 nodes, the energy consumptions per packet were $2.0 \mathrm{~mJ}, 3.0 \mathrm{~mJ}$ and $4.5 \mathrm{~mJ}$ for the transmission ranges of $200 \mathrm{~m}$, $250 \mathrm{~m}$ and $300 \mathrm{~m}$ respectively. The energy consumption per packet was increased by $30 \%$ and $33 \%$ as the transmission range was increased from $200 \mathrm{~m}$ to $250 \mathrm{~m}$ and $250 \mathrm{~m}$ to $300 \mathrm{~m}$ respectively. The energy consumptions per packet were $3.5 \mathrm{~mJ}, 5.5 \mathrm{~mJ}$ and $9.2 \mathrm{~mJ}$ for transmission ranges of $200 \mathrm{~m}, 250 \mathrm{~m}$ and $300 \mathrm{~m}$ respectively for a network consisting of 300 mobile nodes. Hence the energy consumption per packet was increased by almost $50 \%$ as the transmission range was increased from $200 \mathrm{~m}$ to $250 \mathrm{~m}$ and the same was increased by almost $60 \%$ as the transmission range was increased from $250 \mathrm{~m}$ to $300 \mathrm{~m}$. Based on 
the energy consumptions depicted in Fig. 7, we can conclude that the transmission power level should be kept as minimum as possible to save battery of a mobile node. If a network is deployed with the objective to maximize network life, the transmission ranges of mobile nodes should be kept as low as possible.

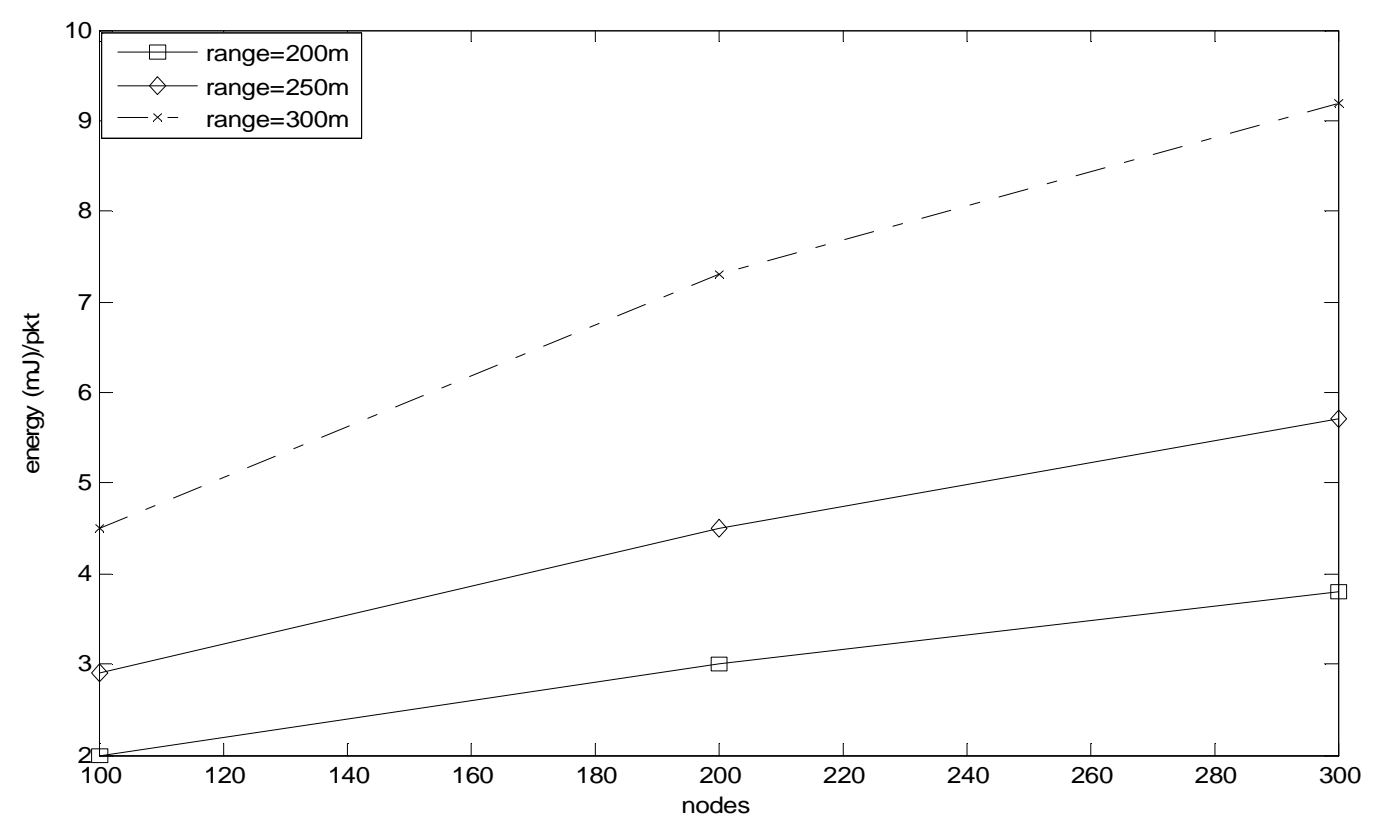

Fig.7 Energy consumptions per packet for different transmission ranges.

\subsection{MAC Overhead}

The MAC overhead packets generated in a network consume a significant portion of node energy (see Fig.6) especially for a large network. If the number of MAC packets generated in a network is reduced, a considerable portion of a node's energy can be saved provided normal functions of MAC layer are not affected. Hence MAC layer packets cannot be reduced arbitrarily. The number of neighbors in a given region of a network is one of the factors that determine the number of MAC packets generated in a network. To reduce the number of MAC packets the transmission range of a mobile node needs to be lowered. If the transmission range is reduced, the number of mobile nodes in a given region will be reduced and hence there will be less number of MAC packets exchanged among the neighbors.

In order to investigate the number of MAC packets generated under different transmission ranges a network consisting of 200 nodes was created and simulated by NS-2. These 200 mobile nodes were deployed over an area of $1000 \mathrm{~m} \times 1000 \mathrm{~m}$. Ten CBR connections were set up randomly in the network. Ten different topologies were tested. The network area was then increased to $1000 \mathrm{~m} \times 1500 \mathrm{~m}$ and $2000 \mathrm{~m} \times$ $1000 \mathrm{~m}$ keeping the node density constant. The results of these simulations are summarized and presented in Fig. 8. The parameter investigated in these simulations is the number of MAC packets per data packet. It is the ratio of the total number of MAC packets generated in the network and total number of data packets delivered to the destinations. This figure demonstrates that the number of MAC packets generated in a network can be reduced if the transmission range is reduced. For example, when the network area was $1000 \mathrm{~m} \times 1000 \mathrm{~m}$, the number of MAC packets per data packet was 1155 for the transmission range of $200 \mathrm{~m}$. But the number of MAC packets per data packet was 1250 and 1325 for the transmission ranges of $250 \mathrm{~m}$ 
and $300 \mathrm{~m}$ respectively. The figure also shows that the number of MAC overhead packets per data packet was always higher for higher transmission ranges. For example, when the network area was the largest (i.e., $2000 \mathrm{~m} \times 1500 \mathrm{~m}$ ), the numbers of MAC packets per data packet are 850,920 and 1150 for the transmission ranges of $200 \mathrm{~m}, 250 \mathrm{~m}$ and $300 \mathrm{~m}$ respectively. A similar conclusion can be reached for the other intermediate network sizes.

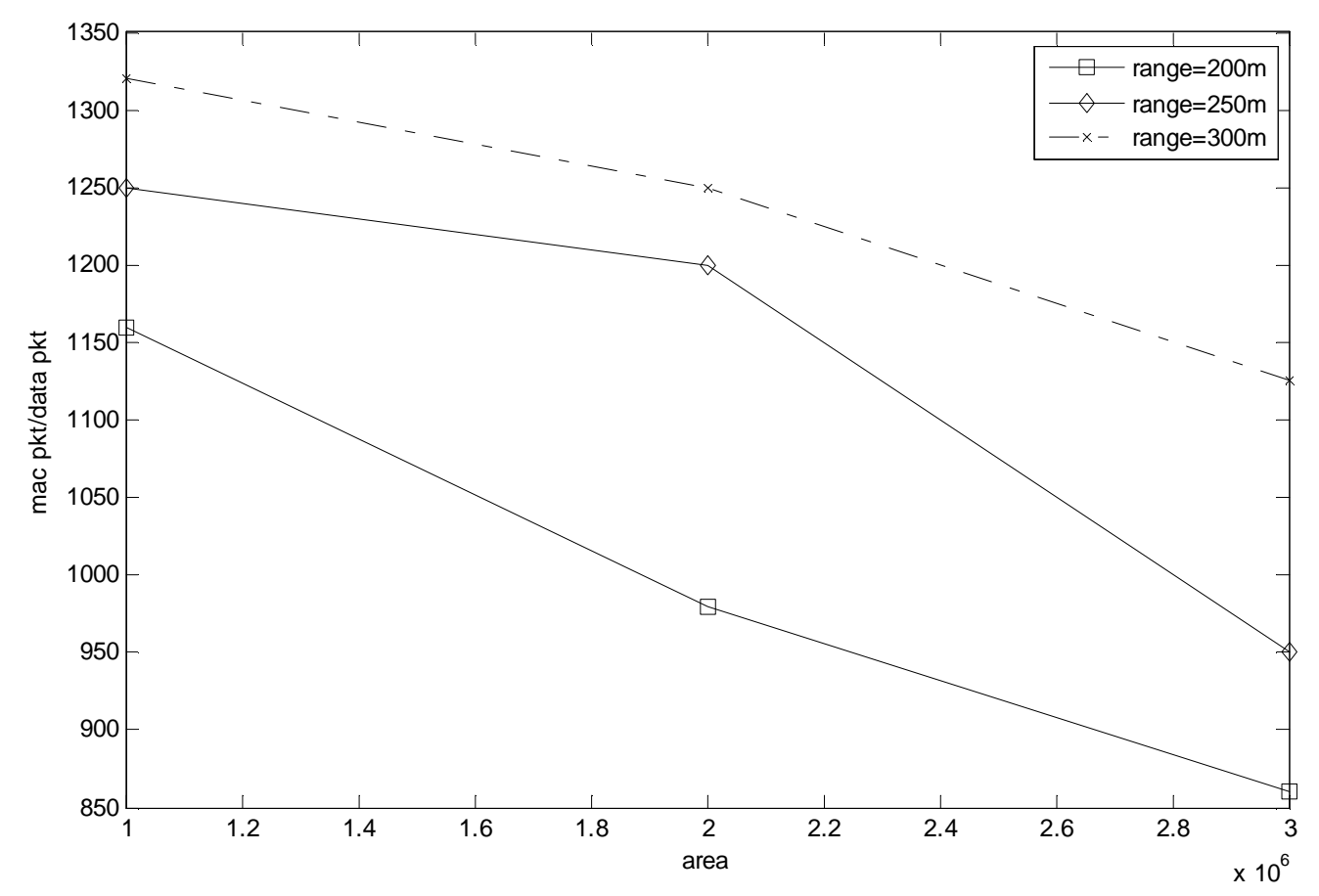

Fig.8 MAC packet generated in the network under different transmission ranges

\subsection{Network Connectivity}

The number of neighbors required to optimize the performance of a network is another issue while selecting short-hop routing and long-hop routing scheme. For a given network, the transmission range of a mobile node determines the number of neighbors in a network. Dependency of network connectivity on the number of neighbors has been investigated by the other researchers as well. The connectivity of a slotted ALOHA [3] based packet radio network has been investigated in [4]. The authors show in [4] that if the number of neighbor is less than six, the network connectivity will be affected and hence the network throughput will be dropped drastically. If the number of neighbor is greater than six, the network throughput also decreases; but at a slower rate. Based on these observations the authors conclude that a mobile node should adjust the transmission power level to a level so that it will have six neighbors. Delay and throughput performances of a network under variable transmission range have been investigated in [5]. The authors also conclude in [5] that mobile nodes should adjust transmission ranges so that they will have eight numbers of neighbors. In this paper, a new parameter called delivery ratio is introduced to indicate the network connectivity. Delivery ratio is defined by the ratio between the number of packets sent to destinations nodes and the number of packet reached these destinations. If network disconnectivity occurs, there will be loss of packets and hence delivery ratio will also drop. On the other hand, if the network is fully connected, the delivery ratio will be high. 
In order to investigate the effects of the transmission range on the delivery ratio for both static and mobility condition, a network consisting of 200 mobile nodes was simulated in NS-2. The mobile nodes were placed randomly over an area of $2000 \mathrm{~m} \times 1000 \mathrm{~m}$ area. Ten connections were set up randomly among the nodes. Each connection used Constant Bit Rate (CBR) agent to generate packet. Once started a CBR agent continues generating packet till the end of the simulation. The number of neighbors $n$ was determined by the following equation

$$
n=\frac{N}{A} x \pi R^{2}
$$

, where $\mathrm{N}$ is the total number of nodes in the network, $A$ is the network area, $R$ is the transmission range. The initial transmission range of a mobile node was set to $200 \mathrm{~m}$. Then the transmission range was increased to higher values. Thus the number of neighbors was varied from 4 to 12 . The delivery ratio performance of the network under varying number of neighbors is depicted in Fig.9. This figure depicts that the delivery ratio improves with the increase in the number of neighbors. A delivery ratio of $100 \%$ was achieved when mobile node had ten neighbors. After this point, the delivery ratio remained constant at $100 \%$. The delivery ratio performance under mobility condition is also shown in Fig. 9. The mobility model used in the simulation is called random-way point model. Random way point model is available with the NS-2 [19]. According to this model, each mobile node moves to a random destination point during the simulation at a preset speed. After reaching the destination point, a mobile node remains there for a certain period of time called 'pause time'. In the simulations, the 'pause time' was set to 200 second and the maximum speed was set to $20 \mathrm{~m} / \mathrm{second}$. When the 'pause time' expires, mobile node again sets a random destination point and starts moving toward that destination point. Since the simulations were run for 250 seconds, we choose the 'pause time' to 200 second to maintain a very low mobility condition in the network. It is depicted in Fig. 9 that the delivery ratio becomes $100 \%$ at 10 numbers of neighbors for a static network. Under mobility condition the delivery ratio also increases with the increase in the number of neighbors. But the delivery ration never reaches at $100 \%$ irrespective of high number of neighbors. A maximum delivery ratio of $98 \%$ was achieved under mobility condition at 8 numbers of neighbors. We can conclude from Fig. 9 that more neighbors are required to achieve the same delivery ratio performance under mobility condition compared to a static network. But there is always packet loss under the mobility condition. Hence $100 \%$ delivery ratio cannot be achieved under a mobility condition.

\subsection{Routing Overhead}

Routing protocol generates a huge number of routing overhead control messages in a network. These control messages are used to collect and maintain information about other mobile nodes located in a network so that necessary routes are discovered and maintained. The number of control messages generated in a network depends upon the type of routing protocol used. In pro-active routing protocol like DSDV [16], mobile nodes periodically exchange routing information among themselves. Hence a huge number of overhead packets are generated in the network. In reactive routing protocol like DSR [17] and AODV [22], mobile nodes exchange routing information on demand. It means mobile nodes exchange routing information only when a mobile node initiates a route discovery process. During the route discovery process, mobile nodes exchange routing information among themselves by using a technique called 'flooding'. According to this flooding technique, a mobile node is obliged to re-broadcast routing 
information that it receives such information from its neighbors. The objective of the flooding is that a source can discover multiple routes to a destination. If one of these routes breaks, a source can use alternative routes. This kind of 'flooding' has little impact on the performance of a small network. But it can adversely affect the performances of a large network. The main problems related to the flooding are (1) contention, (2) collision, and (3) redundancy. A detailed analysis of these problems can be found in [23]. Routing protocols proposed in [23, 24, 25, 26] and [27] reduce the 'flooding' problem. These routing protocols show that the routing overhead can be reduced by controlling flooding. In this paper, we show that the overhead packets can be reduced by controlling the transmission range of a mobile node too. If the transmission range of a mobile node is increased, the route request packet will travel a few number of hops from a source to a destination. Since route request packet travels less number of hops, mobile nodes re-broadcast a request packet a few times. Hence routing overhead will be reduced. On the other hand, if the transmission range of mobile node decreases, the route request packets travel for many hops from a source to a destination. Hence the number of overhead packets will increase.

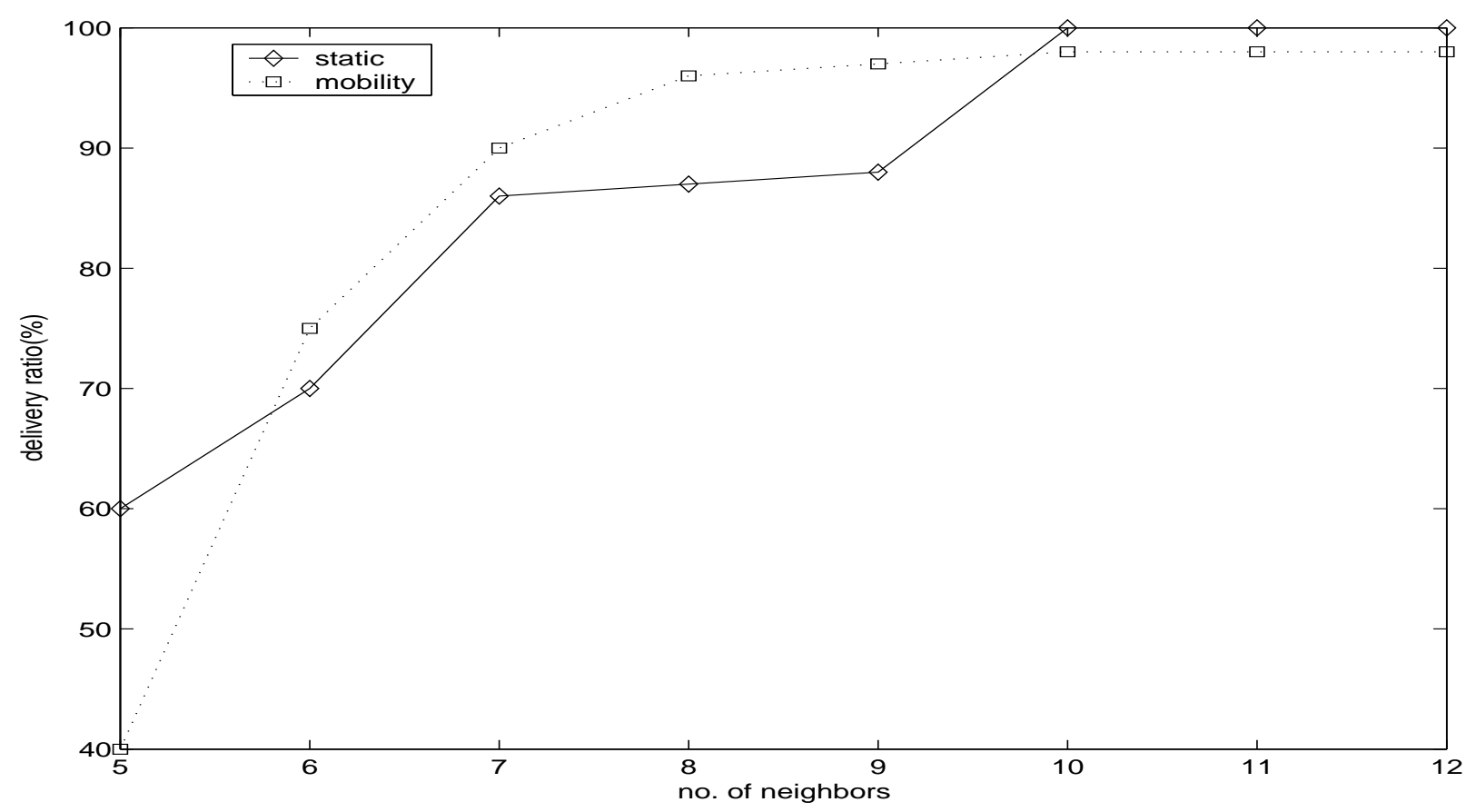

Fig.9 Variation of network connectivity with neighbors

In order to verify this claim, a network consisting of 100 mobile nodes was simulated in NS-2 and the transmission range of a mobile node was varied. The mobile nodes were placed randomly over an area of $1000 \mathrm{~m} \times 1000 \mathrm{~m}$. Ten connections were set up randomly in the network. While setting up each connection, each source initiates a route discovery process. Once a connection is set-up, Constant Bit Rate (CBR) agent was used to generate packets and the packet generation rate was 2 packets/second. Each simulation was run for 250 seconds (simulation time). IEEE 802.11 layer was used as the MAC layer. Since the number of overhead packets generated in a network depends upon the network size, we increased the network size by keeping the node density constant. That means when the network area was increased to $1500 \mathrm{~m} \times 1000 \mathrm{~m}$, we placed 150 mobile nodes in the network to maintain the node density at constant level. Similarly, we placed 200 mobile nodes when the network area was increased to $2000 \mathrm{~m} \times 1000 \mathrm{~m}$. The total number of overhead control packets generated in the network and the total number of data packets reached 


\section{Ml Macrothink}

the destinations were monitored during each simulation. The overhead per data packet was determined based on these two parameters. This is the ratio of the total routing overhead packets generated in the network and the total number of data packets delivered to the destinations. Ten different topologies were created by using random variables. Then this experiment was repeated by using three different transmission ranges of $200 \mathrm{~m}, 250 \mathrm{~m}$ and $300 \mathrm{~m}$. The results are shown in Fig. 10. The figure shows that the overhead packet generated in a network decreases with the increase in transmission range. But for small network the overhead per data packet is almost same irrespective of different transmission ranges. For example, overhead per data packet is almost 0.2 for the transmission ranges of $200 \mathrm{~m}, 250 \mathrm{~m}$ and $300 \mathrm{~m}$ for the smallest simulated network consisting of 100 mobile nodes. But for a larger network (i.e., network consisting of 200 nodes), the overhead per packet are 0.45 and 0.55 for transmission ranges of $250 \mathrm{~m}$ and $200 \mathrm{~m}$ respectively. Hence there was a 10 percent reduction of overhead packets. But the difference in the overhead packet becomes more visible as the network size was further increased. For example, when the network size was the largest (i.e., the network now has 300 mobile nodes) there was a significant amount of decrease in the number of overhead packets as the transmission range was reduced. Fig.10 shows that the overhead packets were reduced by $20 \%$ as the transmission range was decreased from $250 \mathrm{~m}$ to $200 \mathrm{~m}$. The overhead was further reduced by $30 \%$ as the transmission range was further reduced by $50 \mathrm{~m}$. The reason for this kind of overhead reduction is that the number of neighbors in a given region decreases as the transmission range is lowered. Hence there is less number of neighbors that participate in the route discovery process and there will be less number of re-broadcasting of request messages. But the number of neighbors in a given region increases with the increase in the transmission range. Hence a large number of mobile nodes that participated in the route discovery process and higher overhead control packets are generated in the network.

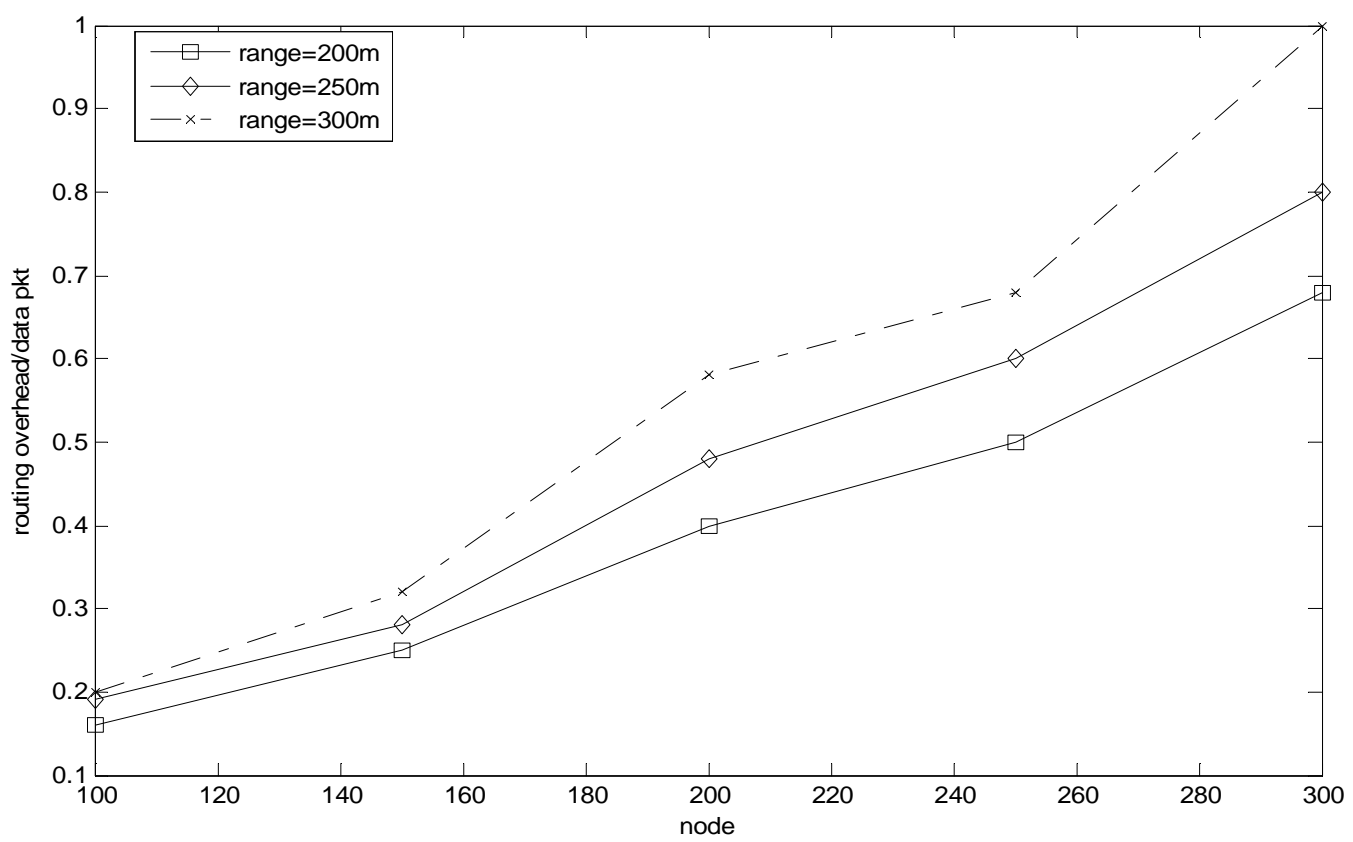

Fig.10 Routing overhead generated with varying transmission range

\subsection{End-to-End Delay}

The end-to-end delay per packet is the time taken by a data packet to travel from its source of origin to 
its destination. This traveling time is influenced by two major factors (1) the number of hops that a packet travels, and (2) how much busy is the medium around each hop. After receiving a packet, a mobile node needs to get access to the medium. The access method depends on what type of medium access mechanism is used. If medium access control algorithm like CSMA/CA (i.e., 802.11) is used, a mobile node is allowed to transmit a packet if it finds the surrounding medium is free. Otherwise, a mobile node needs to wait for a random period of time. This waiting time of each node also depends on how many mobile nodes are there in a given region and how much traffic each of these mobile nodes is carrying. If the transmission range is low, a packet will travel many short-hops. Hence a packet needs to wait at many hops and it takes longer time to travel. On the other hand, if long-hop routing is used, a packet needs to wait at few numbers of hops. To investigate this issue, a network consisting of 100 nodes was created in NS-2. The mobile nodes were placed over area of $1000 \mathrm{~m} \times 1000 \mathrm{~m}$. Ten different CBR connections were set up in the network. The simulation results are shown in Fig. 11. This figure shows that the end-to-end delay per packet depends on the transmission range of a mobile node. A low transmission range increases the end-to-end delay per packet. But the variation in the end-to-end delay with the transmission range is not significant for a small network. The reason is that a packet travels only a few hops (i.e., one or two) from a source to a destination in a small network. But the delay variation is more significant for a large network. For example, when the network size was medium (i.e., 200 nodes) and the transmission range was increased from $200 \mathrm{~m}$ to $250 \mathrm{~m}$; the delay per packet was reduced by $20 \%$. This delay was further reduced by $30 \%$ as the transmission range is further increased from $250 \mathrm{~m}$ to $300 \mathrm{~m}$. For a network size of 300 nodes, there is a reduction in delay of $40 \%$ as the transmission range is increased from $200 \mathrm{~m}$ to $250 \mathrm{~m}$. The figure also shows the delay per packet is further reduced by $30 \%$ when the transmission range is further increased from $250 \mathrm{~m}$ to $300 \mathrm{~m}$.

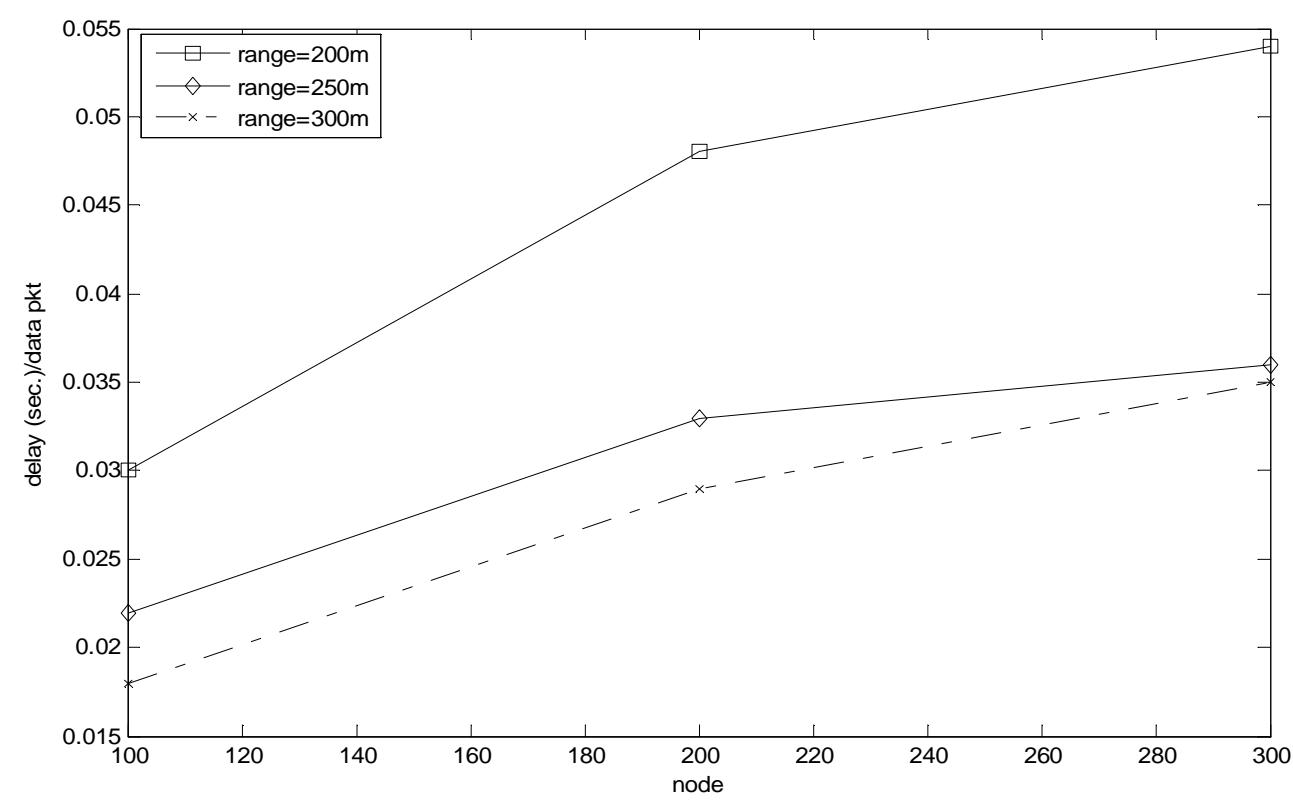

Fig.11 End-to-end delay comparison

\subsection{Packet loss}

Packet loss is another important parameter that affects the performance of a MANET. It is very crucial 
issue for MANETs that are deployed for multi-media applications as well as for defense applications. Three major reasons for packet loss are (1) packet dropped due to limited buffer size of a mobile node, (2) packet loss due to packet collision, and (3) packet was received at a signal level that is less than a threshold value. Each mobile node has a buffer of limited size. After receiving a packet, a mobile node keeps the packet in the buffer for a temporary period of time. Packets wait there until mobile node gets access to the medium to transmit that packet. If a mobile node handles too much traffic, there is high probability of packet loss due to limited buffer size. It is shown in [5] that controlling transmission range of a mobile node can reduce packet loss due to limited buffer. Another cause of packet loss is packet collision. Short-hop routing has gained a lot of support to reduce packet loss in a network due to packet collision. The packet collision probability for IEEE 802.11 MAC layer has been formulated in [23], which is given by equation 5 .

$$
\gamma=1-e^{-(n-1) \alpha}
$$

, where $n$ is the number of neighbors in a given region, $\alpha$ is the parameter of the exponential back-off duration and this $1 / \alpha$ has units of time. When the transmission range is low, the number of neighbors in a given region will decrease. Hence the packet loss will be reduced. However, the parameter $\alpha$ depends on how much traffic is there in a given region of a network. Another reason of packet loss is due to weak signal strength. It is mandatory that packet should be received at a mobile node with a power (i.e., received power) that should be more than a threshold value. Otherwise, a mobile node cannot correctly decode the information content of a packet. The received power varies with the distance between two nodes and expressed by the following equation:

$$
P_{r} \alpha \frac{1}{d^{n}}
$$

, where $d$ is the distance the packet has traveled and $n$ is a path loss exponent. The value of $n$ varies between 2 to 4 depending upon the locations. Short-hop routing has gained a lot of support to reduce packet loss due to poor received power. The reason is that when a packet travels small distance, the probability of receiving the packet at a power level above the threshold value is high. Hence there is less chance to lose a packet due to weak signal level. On the other hand, if long-hop routing is used, there is a high probability that the signal strength will go below a threshold value. Hence there will be more packet loss in the network.

In order to investigate the packet loss probability, a network consisting of 200 nodes was created. The mobile nodes were placed over an area of $2000 \mathrm{~m} \times 1000 \mathrm{~m}$. Ten CBR connections were set up in the network. To test the packet loss probability due to high traffic intensity, we increased the packet generation rate. The delivery ratio performance is illustrated in Fig. 12. It is shown in this figure that there will be very negligible packet loss in the network if there is not too much traffic in a network. The delivery ratio is almost $100 \%$ up to packet generation rate of 4.0 packets/sec. After this point, the delivery ratio decreases rapidly. The delivery ratio remains high for the low transmission range (i.e., 200m). 


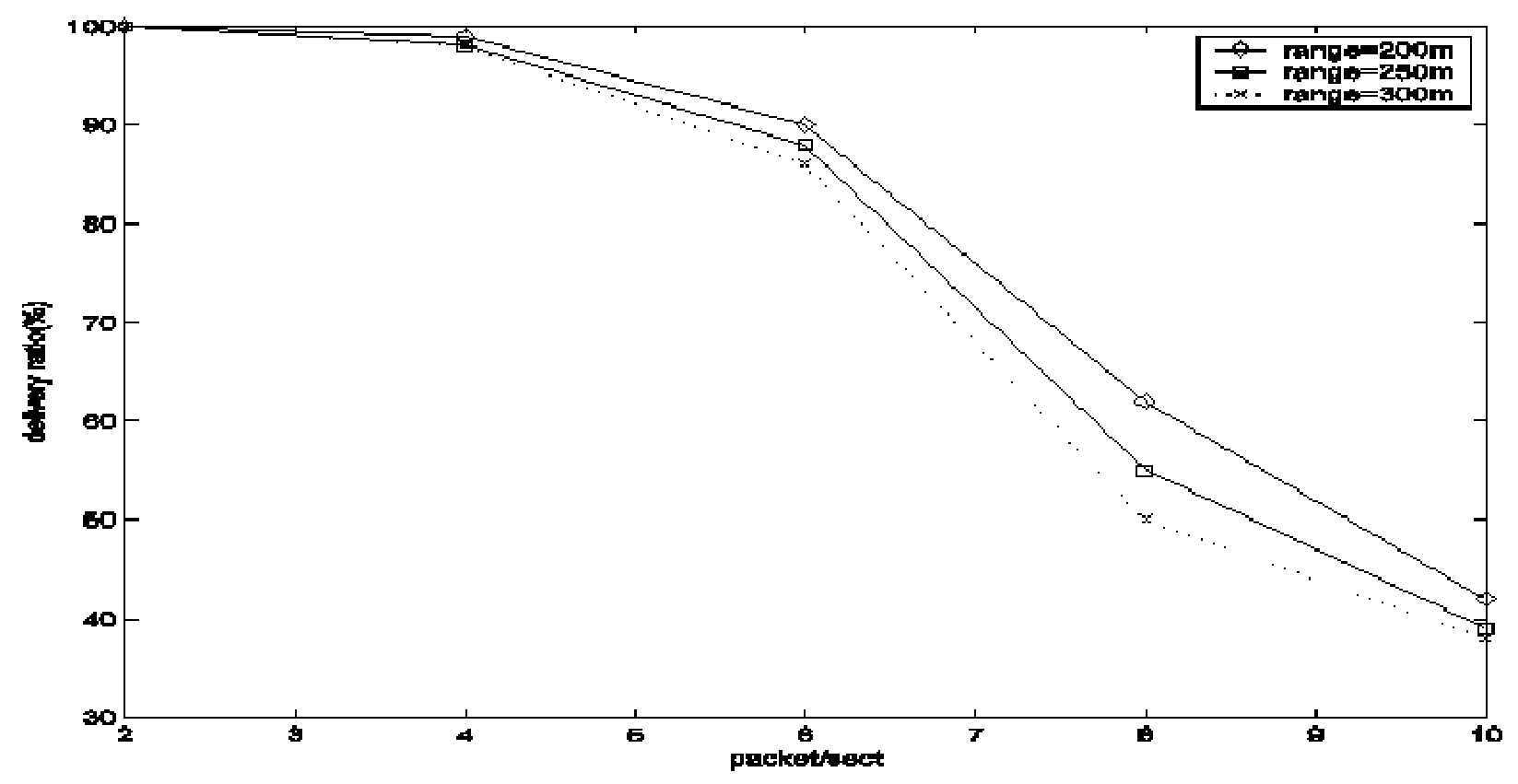

Fig.12 Delivery ratio performance

\subsection{Mobility}

Dynamic topology is an inherent characteristic of MANET. Mobile nodes are free to move at any time and at any direction. Network performances such as packet loss and hence the network throughput is also related to the mobility condition in a network. Since communication in MANETs is of multi-hop nature, route 'breaks' when an intermediate hop moves out of the reach of previous hop. Route breakage is handled with the route maintenance mechanism of reactive routing protocol. When a route breakage occurs, some packets are lost because these packets do not have any route available to travel. Hence packet loss in the network will increase with the increase in the mobility of the nodes. To test the effects of mobility on packet loss, a mobility model called random waypoint model was again used. To test the performances of a network under different mobility conditions, a network consisting of 200 nodes was created. The mobile nodes were placed over an area of $2000 \mathrm{~m} \times 1000 \mathrm{~m}$. The mobile node's speed was set to $20 \mathrm{~m} / \mathrm{second}$. The transmission ranges were varied and the network was tested again for different topologies. The pause time was also varied to investigate the network performance under different mobility condition. The initial pause time was set to 200 second. Then the pause time was decreased to 150 second and 100 second. The simulations were run for 250 second. The pause time of 100 second means the highest level of mobility condition in the network. The results of the simulations are shown in Fig.13. It is depicted in this figure that the delivery ratio is high when the transmission range is high. Using higher transmission range a mobile node takes longer time to move out of the reach from its neighbor. On the other hand, a mobile node quickly moves out of the reach of its neighbor if transmission range is low. Fig. 13 also shows that the delivery ratio is almost $95 \%$ when the network has the least amount of mobility (i.e., pause time is 200 second). For higher mobility condition (i.e., pause time is 100 second), the delivery ratio is $30 \%$ for the transmission range of $200 \mathrm{~m}$. The delivery ratio improves with the increase in transmission range. For example, the delivery ratios were $35 \%$ and $45 \%$ for the transmission ranges were $250 \mathrm{~m}$ and $300 \mathrm{~m}$ respectively. We can conclude from the simulation results that the long-hop routing is suitable for high mobility condition. On the other hand, under a low mobility condition transmission range has a little impact on the delivery ratio. 


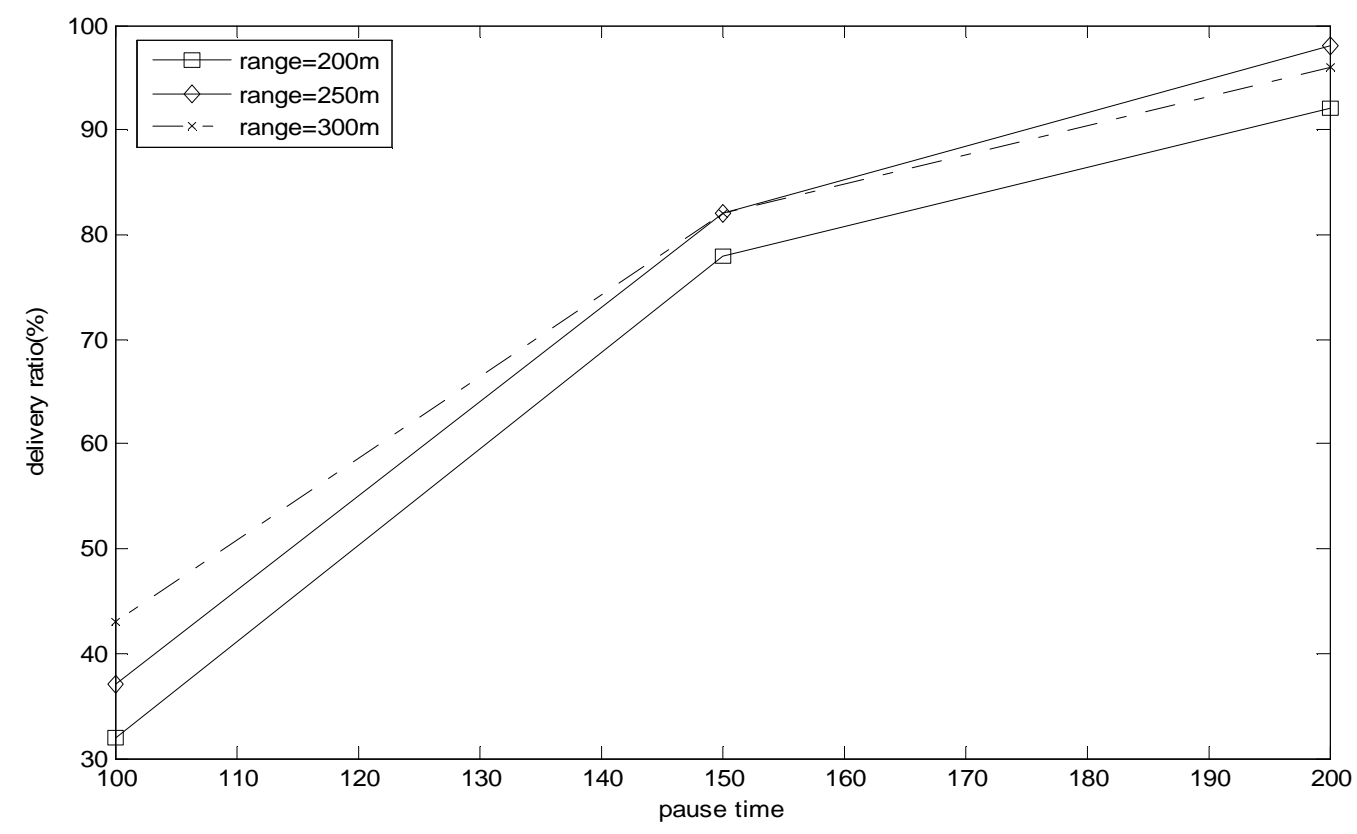

Fig.13 Delivery ratio under mobility condition

\section{CHOOSING SHORT-HOP OR LONG-HOP ROUTING}

From the simulation results presented so far in this paper we can conclude that long-hop routing and short-hop routing have their own advantages and disadvantages. While choosing the right one between these two routing algorithms one should consider the intended application of MANETs. MANETs typically require multihop routing since it covers a large area and the mobile node has limited transmission range. One of the main challenges of MANET is to support high data rate over multiple hops where link quality of each hop is different and changes over time. The lack of a centralized administration and high mobility of users are the major obstacles in attaining high network throughout. From the network throughput investigations it is evident that long-hop and short-hop routing are directly related to the network throughput. Fig. 4 shows that a maximum throughput was achieved when the transmission range was the maximum (i.e., range 250 meter). This maximum throughput was achieved at the packet generation rate of 5 packets/second. But the network throughput decreased very quickly for higher packet generation rate. A more sustainable throughput was achieved at the lower transmission ranges. Hence the multimedia applications that need higher throughput the short-hop routing scheme is a better choice. On the other hand, long-hop is a better choice for medium throughput. The quality of a link between a transmitter and receiver is an important issue for MANETs. The quality of link is usually measured by Signal-to-Interference and Noise (SNR) ratio. The SNR varies with time because the signal travels through a wireless medium that is always changing with respect to time. This kind of SNR variation results in shadowing effects. The shadowing effects explain the reason why the signal level may go below a threshold level and a receiver cannot detect this signal at this level for a given transmitter-receiver distance. This kind of signal variations causes packet loss in a network. It is shown in this paper that a careful selection of transmission range is required to cope with this shadowing effects. Fig. 5 compares the packet losses due to shadowing effects under different transmission ranges. This figure shows that transmission range should be kept as high as possible to reduce packet loss in a network. In mission critical applications (i.e., war field) and industrial 
process control applications, packet loss should be kept as low as possible. Hence in these applications long-hop routing is preferable.

Energy consumption is one of the important design issues for energy constraint applications. The battery of mobile node needs to be operative as long as possible. This is a critical issue for a mobile node whose battery cannot be replaced or recharged. Mobile node placed in the concrete to monitor structural strength is an example for such application. Routing protocols have been proposed to minimize energy consumption in MANET. These protocols use different approaches to minimize energy consumption. These approaches can broadly be classified in three categories: (1) transmit power control [29, 30, 31, 32, 33] and [34]; (2) load distribution [35] and [36]; and (3) sleep/power down [37] and [38]. In load distribution approach, network traffic is distributed among the mobile nodes so that mobile nodes are not over utilized or under -utilized. In sleep-power down approach, some mobile nodes are put into sleep mode to conserve battery power. In the last approach, the transmission power of mobile node can be reduced, so that mobile node spends less amount of energy while transmitting a packet. Fig. 7 shows that the energy consumption can be significantly reduced if short-hop routing is used. Even if the transmission range is reduced by only 50 meter, almost $50 \%$ energy of mobile node can be reduced. But energy consumption may not be a major issue if the mobile node is attached to a very large power source. For example, a mobile node located in an automobile may be attached with a large energy source. In this case, long-hop routing and short-hop routing in not an important design issue. Maintaining network connectivity is another important issue for a MANETs. MANETs are dynamic topologies. Mobile nodes always move into or out the network at any time. This kind of movement causes route breakages in a network. Hence packets are lost due to route breakage. The number of mobile nodes located in a given region (i.e., neighbors) is therefore important for MANETs. If there are higher number of neighbors, there will exist more number of paths for a given source-destination pair. Hence if one route is 'broken', alternative route will be available to continue communication. Fig. 9 shows clearly the effects of number of neighbors on the network connectivity. It shows that more neighbors are required to improve network connectivity. But an arbitrary increase in the number of neighbors did not ensure 100\% connectivity. On the other hand, for static network, the less number of neighbors are required to ensure $100 \%$ delivery ratio. But long-hop routing is a better choice under mobility condition. Categorical energy consumptions by different types of packet were presented in Fig. 6. It is depicted there that the energy consumptions by different types of packets depend upon the network size. When the network size is large, a significant portion of mobile node's energy is spent in transmitting overhead packets. The routing overhead and Medium Access Control (MAC) overhead packets are the major components of total overhead packets generated in MANET. The routing overhead analysis (Fig. 10) shows that long-hop routing reduces the number of routing overhead control packets generated in a network. Less routing overhead control packets is highly desirable for MANETs. Because a huge number of redundant overhead control messages generated in a network during the route discovery phase of the protocol affect the performance of a network. By reducing overhead control messages, network resources like bandwidth can be used more efficiently for transmitting useful data packet. The MAC overhead packet also consumes a significant portion of node's energy. Hence the number of MAC overhead should be kept at a minimum level. The MAC overhead packets in a network can be reduced if the number of node in a given region is reduced. By reducing the transmission range, the number of MAC packets can be reduced as shown in Fig. 8. Since the MAC overhead packets and the routing overhead packets are related to transmission range, the energy consumption can be reduced by reducing the overhead control packets. In this way mobile nodes can conserve energy. Hence mobile 
nodes will be left with more energy to transmit useful data packet. Quality of Service (QoS) support is critical in wireless home application, video on-demand, audio on-demand and voice over IP applications. Time bounded services like audio and video conferencing typically require some specified delay guarantee. The delay constraint of a network is also related to transmission range of mobile nodes. To ensure network delay performance long-hop routing performs better than short-hop routing as shown in Fig. 11. This figure shows that long-hop routing improves the delay performance irrespective of network size. But the effect of long-hop routing is more effective for a network of large size. Hence long-hop routing should be used if MANETs are deployed to support delay constraint applications. To ensure reliable communication under dynamic topology is another challenging issue of MANETs. Packet loss in a network is one of the major causes of unreliable communication. Mobile nodes temporarily store packets in a buffer and transmits packet as soon as it finds the route for the packet. According to the route maintenance technique of reactive routing protocol, a mobile node tries to find alternative route if the current route breaks. But there is a time gap between the detection of a route failure and the selection of alternative route. During this time gap, mobile nodes loose packets. Another reason of packet loss is that the buffer of a mobile node may be full of packets, which do not have routes yet. These two cases vary with the mobility conditions in the network. If there is more mobility in a network, there will be more packet loss in a network. Fig. 13 shows that long-hop routing reduces packet loss in a network under mobility condition. When long-hop routing is used, packet travels for a few hops from a source to a destination. Hence there is less probability that packet will be lost due to buffer overflow. On the other hand, if short-hop routing is used, packet travels many hops from a source to a destination. Hence there is a high probability to lose packet at each hop. Moreover, long-hop routing ensures less route breakage because a mobile node takes longer period of time to go out of range of the previous hops. Not only that route maintenance mechanism of routing protocol takes less time to detect faulty link and hence takes less time also to make route maintenance actions. The reason is that a route error packet that is used to detect route failure also travels less number of hops. Hence source can select alternative route from the route cache within a short period of time.

\section{CONCLUSIONS}

In this paper, the performances of MANET have been investigated under short-hop and long-hop routing conditions. The simulation results show that it is hard to find an optimum transmission range that will meet all the diversified performance requirements of MANETs such as (1) maximize network capacity, (2) reduce overhead, (3) reduce energy consumption, (4) improve delivery ration and (3) reduce packet loss. But it is shown clearly that the decision whether short-hop routing or long-routing be used depends upon the network conditions and it is application specific. Some of the decision criteria are how many nodes are there, how much traffic a network is carrying, what is the mobility condition in the network, how severe is the shadowing effects, what should be the life-span of the network and how much delay tolerance is needed. If the main objective of a network is to reduce the delay per packet, long-hop routing performs better. But long-hop routing consumes more energy. Hence it will reduce network life. So if the objective of a network is that it should be kept operative a long as possible, short-hop routing is a good choice. When there is more mobility in the network, long-hop routing helps to reduce packet loss. If a network has problem with high overhead packets, long-hop routing is preferable because it produces less overhead in the network. Hence we can conclude that there is no definite answer for the question that when one should use shot-hop routing and when one should use long-hop routing. One should choose either long-hop routing or short-hop routing depending upon the network conditions, performance objectives and 
applications of the network.

In order to investigate the effects of short-hop or long-hop routing scheme on the performances of MANETs a wide variety of performances parameters were investigated in this paper. The main reason for choosing these performance parameters is to draw the attentions of the interested researchers to do further in depth investigations on these issues. For example, the shadowing effects have been investigated thoroughly in [39]. Similarly, other effects can be investigated in more details to come up with a concrete conclusion about choosing the suitable routing scheme.

\section{References}

[1] Theodore S. Rappaport, Wirelesss Communication: Principle and Practice, Second Edition, Prentice-Hall Inc., Upper Saddle River, New Jersy, USA, pp. 138-140

[2] J. Jubin and J. Tornow, The DARPA Packet Radio Network Protocols, In the Proceedings of the IEEE, 75(1), 1987, pp. 21-32

[3] D. Bertekas and R. Gallager, Data Networks, Prentice Hall, Upper Saddle River, NJ 07458, Second Edition, 1992, pp. 349-350

[4] L. Kleinrock and J. Silvester, Optimum transmission Radii for packet Radio Networks or why six is a Magic Number, In the Proceedings of the IEEE National Telecommunication Conference, Birmingham, Alabama, December 1978, pp. 4.3.1-4.3.5

[5] H. Takagi and L. Kleinrock, Throughput Delay Characteristics of Some Slotted-aloha Packet Radio Networks, IEEE Transaction on Communication, Vol. 33, 1985, pp. 1200-1207

[6] M. Shanchez, P. Manzoni and Z.J. Haas, Determination of Critical Transmission Range in Ad hoc Networks, In the Proceedings of the International Conference on New Technologies, Mobility and Security (NTMS), Paris, France, May 2-4, 2007, pp. 1-12

[7] T.A. Elbatt, S.V. Krishnamurthy, D. Connors, and S. Dao, Power Management for Throughput Enhancement in Wireles Ad hoc Networks, In the Proceedings of the IEEE International Conference on Communications (ICC), New Orleans, LA, June 2000, pp. 1503-1513

[8] E. M. Royer, P. Micheal, M. Smith and L. E. Moser, An analysis of the Optimum Node Density for Ad hoc Mobile Networks, In the Proceedings of the International Conference on Communication (ICC), Helsinki, Finalnd, June 11-14, 2001, Vol.3, pp. 857-861

[9] M.Haenggi and D. Puccinelli, Routing in Ad Hoc Networks: A case of long hops, IEEE Communication Magazine, October 2005, pp. 93-101

[10] M. Tarique and K. E. Tepe, A New Hierarchical Design for Wireless Ad hoc Network with Cross Layer Design, International Journal of Ad hoc and Ubiquitous Computing, Vol. 2, No. 1/2, 2007, pp. 12-21

[11] T.A. ElBatt, S.V. Krishnamurthy, D. Connors, S. Dao, Power Management for Throughput Enhancement in Wireless Ad-Hoc networks, IEEE ICC'00, New Orleans, LA, June 2000

[12] R. Ramanathan, R. Rosales-Hain, Topology Control of Multihop Wireless Networks using Transmit Power Adjustment, Procedings of INFOCOM 2000

[13] R. Wattenhofer, L. Li, P. Bahl, and Y.-M. Wang, Distributed topology control for power efficient operation in multihop wireless ad hoc networks, in Proceedings of INFOCOM, 2001, pp. $1388-1397$

[14] Kawadia V., Kumar P.R., Power Control and Clustering in Ad Hoc Networks, IEEE INFOCOM, 
April 2003.

[15] K. Manononsakis and A. J. AcAuley, “ Minimum necessary transmission range assignment for network connectivity", In the Proceedings of the $18^{\text {th }}$ Annual IEEE International Symposium on Personal, Indoor and Mobile Radio Communication (PIMRC), Athens, Greece, September 2007, pp. $1-5$

[16] C.E. Perkins and P. Bhagwat, Highly dynamic destination-sequence distance-vector routing for mobile computers, In the Proceedings of ACM SIGCOMM, London, United Kingdom, August 1994, pp. 234-244

[17] J. Broch, D. B. Johnson, and D. A. Maltz, The Dynamic Source Routing Protocol for Mobile Ad Hoc Networks, IETF Internet-Draft, draft-ietf-manet-dsr-00.txt, March 1998

[18] V. Bharghavan, A. Demers, S. Shenker, and L. Zhang, MACAW: A media access protocol for wireless $L A N s$, In the Proceedings of ACM SIGCOMM, London, UK, August 1994, pp. 212-225

[19] K. Fall and K. Varadhan, NS Notes and Documentation Technical Report, University of California Berkeley, LBL, USC/ISI, and Xeron PARC

[20] S. Doshi, S. Bhandare and T.X. Brown, An On-demand Minimum Energy Routing Protocol for Wireless Ad hoc Network, The ACM SIGMOBILE Mobile Computing and Communication Review, Vol. 6, No. 2. pp. 50-66

[21] L.M. Feeny and M. Nilsson, Investigating energy consumption of wireless network interface in ad hoc networking environment, In the Proceedings of the Twentieth Annual Joint Conference of the IEEE Computer and Communications Societies (INFOCOM 2001), Vol. 3, April 2001, pp. $1548-1557$

[22] C.E. Perkins, Ad Hoc On Demand Distance Vector (AODV) routing, IETF Internet-Draft, draft-ietf-manet- aodv-00.txt, November 1997

[23] S.Y. Ni, Y.C. Tseng, Y. S. Chen, and J. P. Sheu, The Broadcast Storm Problem in a Mobile Ad Hoc Networks, MobiCom99, Seattle, Washington, August 15-20 1999, pp. 151-162

[24] B. Krishnamachari , S.B. Wicker, and R. Bejar, Phase transition phenomenon in wireless ad-hoc networks, In the Proceedings of GLOBOCOM, San Antonio,Texas, November,2001, pp. 2921-2915

[25] Y. Sasson, D. Cavin and A. Schiper, Probabilistic Broadcast for flooding in Wireless Mobile Ad hoc Networks, Swiss Federal Institute of Technology, Switzerland, Technical Report IC/2002/54

[26] J. Z. Haas, Y. J. Halpern, and L. Li, Gossip Based ad hoc routing, In the Proceedings of IEEE INFOCOM 2002, New York, Volume 3, June 2002, pp. 1707-1716

[27] H. Lim and C. Kim, Multicast tree construction and flooding in wireless ad hoc networks, In he Proceedings of ACM International Workshop on Modeling, Analysis and Simulation of Wireless and Mobile Systems,Boston,2000

[28] K., Anurag, d. Manjunath and j. Kuri, Communication Networking: An Analytical Approach, Morgun Kauffman Publishers, San Francisco, CA, USA, pp. 715-716

[29] J-H. Chang and L. Tassiulus, Energy conserving routing in Wireless Ad Hoc Networks, In the Proceedings of IEEE INFOCOM, March, 2000, pp. 22-31

[30] Q. Li, J. Aslam and D., Rus Online power aware routing in Wireless Ad Hoc Networks, In the Proceedings of the seventh International Conference on Mobile Computing and Networking (MobiCom), July, 2001, pp. 97-107

[31] L. Stojmenovic and X. Lin Power aware localized routing in wireless networks, IEEE Transaction 


\section{MInstitute Macrothink $_{\text {Int }}$}

on Parallel and Distributed Systems, Vol. 12, No. 11, November,2001, pp. 1122-1133

[32] S. Doshi, S. Bhandare and T.X. Brown An On-demand minimum energy routing protocol for wireless ad hoc network, The ACM SIGMOBILE Mobile Computing and Communication Review, Vol. 6, No. 2, pp. 50-66

[33] S. Benerjee and A. Misra Minimum energy path for reliable communication in multi-hop wireless network, In the Proceedings of the third ACM Annual workshop on Mobile Ad Hoc Networking and Computing (MobiHoc), June, 2002, pp. 146-156

[34] S. Narayanaswamy, V. Kawdia, R.S. Srinivas and P.R. Kumar Power Control in Ad Hoc Network: Theory, architecture, algorithm and implementation, In the Proceeding of European Wireless Conference -Next generation wireless Networks, Technologies, Protocols, Services and Applications, February, 2002, pp. 156-162

[35] K. Woo, C. Yu, H.Y. Youn and B. Lee Non-Blocking, localized routing algorithm for balanced energy consumption in mobile ad hoc networks, In the Proceedings of the International Symposium on Modeling, Analysis and Simulation of Computer and Telecommunication System (MASCOTS), August, 2001, pp. 117-124

[36] C.K. Toh Minimum battery life to support Ubiquitous Mobile Computing in Wireless Ad Hoc Networks, IEEE Communication Magazine,Vol. 39, No. 6, June, 2001, pp.138-147

[37] B. Chen, K. Jameison, R. Morris, H. Balakrishnan SPAN: An energy Efficient Coordination Algorithm for Topology Maintenance in Ad Hoc Wireless Networks In the Proceedings of the sixth Annual Conference on Mobile Computing and Networking (Mobicom), July, 2001, pp. 85-96

[38] Y. Xu, J. Hiedemann and D. Astrin Geogrpahy-informed energy conservation for ad hoc routing, In the Proceedings of the seventh Annual International Conference on Mobile Computing and Networking (Mobicom), July, 2001, pp. 70-84

[39] Md. Anwar Hossain, Mohammed Tarique and Rumana Islam," Shadowing Effects on Routing Protocol of Multihop Ad Hoc Networks", International Journal of Ad hoc, Sensor and Ubiquitous Computing, Vol. 1, No. 1, March 2010, pp. 12-28 